\title{
VII. Oxígenoterapia y rehabilitación respiratoria en el paciente con enfermedad pulmonar obstructiva crónica
}

JUAN CÉSPEDES G.*,** y FRANCISCO ARANCIBIA H.*,***

\author{
Oxygen therapy and pulmonary rehabilitation in patients with chronic obstructive \\ pulmonary disease
}

Patients with chronic obstructive pulmonary disease (COPD) often have severe shortness of breath that prevents them from performing their everyday activities. Pulmonary rehabilitation programs improve capacity exercise, decrease breathlessness and enhance quality of life. In addition, the use of oxygen has been demonstrated to reduce ventilatory demand and to induce improvements in metabolism, muscle function, and cardiovascular function. The combination of exercise training and supplemental oxygen may provide additional benefit. This chapter therefore evaluated the scientific evidence regarding the beneficial effect of supplemental oxygen in the pulmonary rehabilitation of patients with COPD. Supplemental oxygen should be recommended in all COPD patients with resting hypoxemia during rehabilitative exercise training (quality evidence A, strong recommendation). Also, in COPD patients without resting hypoxemia was recommended to use supplemental oxygen during respiratory rehabilitation, because it allows a longer time of exercise and reduces dyspnea (quality evidence B, weak recommendation).

Key words: Supplemental oxygen, respiratory rehabilitation, COPD, resting hypoxemia.

\section{Resumen}

Los pacientes con enfermedad pulmonar obstructiva crónica (EPOC) a menudo tienen dificultad respiratoria severa que les impide realizar sus actividades cotidianas. Los programas de rehabilitación pulmonar mejoran la capacidad de ejercicio, disminuyen la disnea y mejoran la calidad de vida. En adición, el uso de oxígeno ha demostrado una reducción de la demanda ventilatoria y mejorías en el metabolismo, la función muscular y la función cardiovascular. La combinación de entrenamiento muscular y suplemento de oxígeno puede proporcionar un beneficio adicional. En este capitulo se evaluó la evidencia científica que existe en cuanto al beneficio del uso del suplemento de oxigeno en la rehabilitación respiratoria en pacientes con EPOC. Se recomendó el uso de suplemento de oxígeno en todo paciente con EPOC e hipoxemia de reposo durante el entrenamiento muscular (calidad de la evidencia A, recomendación fuerte). Así también, en los pacientes con EPOC y sin hipoxemia de reposo se recomienda utilizar suplemento de oxígeno durante la rehabilitación respiratoria, porque permite tolerar durante mayor tiempo el entrenamiento muscular y reduce la disnea (calidad de la evidencia $B$, recomendación débil).

Palabras clave: Oxígeno suplementario, rehabilitación respiratoria, EPOC, hipoxemia de reposo.

\section{Introducción}

Los pacientes con enfermedad pulmonar obstructiva crónica (EPOC) pueden presentar hipoxemia significativa en reposo, especialmente en los estadios GOLD III y IV. No obstante, estos pacientes en todos los estadios de la enfermedad pueden tener desaturación durante la actividad física, lo que provoca no sólo disnea sino también limitación importante para realizar las actividades de la vida diaria y por ende un deterioro de su calidad de vida ${ }^{1}$.

\footnotetext{
* Servicio de Medicina Respiratoria, Instituto Nacional del Tórax.

** Servicio Broncopulmonar, Clínica Los Coihues.

***Unidad de Respiratorio, Clínica Santa María.
} 
La hipoxemia aumenta la ventilación pulmonar mediante la estimulación directa de los quimiorreceptores periféricos y secundariamente al hacer ejercicio por la formación de ácido láctico proveniente del metabolismo anaeróbico. La lactacidemia asociada con el aumento de la producción de $\mathrm{CO}_{2}$, contribuyen en aumentar la ventilación al actuar como un potente estímulo a nivel del quimiorreceptor central ${ }^{2}$.

El aumento de la ventilación en estos pacientes se hace mayoritariamente a expensas de la frecuencia respiratoria, lo que favorece la hiperinsuflación dinámica y atrapamiento aéreo, con desplazamiento de la curva de distensibilidad hacia la izquierda y aparición de disnea por el aumento del trabajo respiratorio y la mayor necesidad de fuerzas inspiratorias².

Las alteraciones señaladas anteriormente limitan la capacidad de hacer ejercicio y por ende afectan el resultado obtenido en los programas de rehabilitación respiratoria (RR) al disminuir la capacidad e intensidad del trabajo que los enfermos pueden alcanzar.

La justificación del uso de oxígeno para aumentar la capacidad de ejercicio es que reduce la disnea de esfuerzo. Esto, probablemente a través de varios mecanismos, incluyendo la reducción de la demanda ventilatoria ${ }^{3}$, retrasando la acidemia metabólica del ejercicio, e indirectamente por la reducción de la hiperinsuflación dinámica ${ }^{4}$. Esto último se logra mediante la reducción de la ventilación y la frecuencia respiratoria, lo que permite un tiempo espiratorio más prolongado y un mejor vaciado pulmonar.

\section{Preguntas}

1. ¿La oxígenoterapia durante la rehabilitación respiratoria mejora el rendimiento físico en pacientes con enfermedad pulmonar obstructiva crónica?

2. ¿Los pacientes con EPOC y sin hipoxemia de reposo se benefician de la oxígenoterapia en los programas de rehabilitación respiratoria?

\section{Pacientes}

Pacientes con enfermedad pulmonar obstructiva crónica estable que presentan disnea y limitación de la capacidad de realizar ejercicio a pesar del tratamiento farmacológico óptimo.

\section{Intervención}

Administración de oxígeno durante la realización de entrenamiento muscular de extremidades superiores e inferiores en un programa de rehabilitación respiratoria.

\section{Objetivo}

Evaluar si la oxígenoterapia aplicada durante los programas de rehabilitación respiratoria es beneficiosa en pacientes con enfermedad pulmonar obstructiva crónica.

\section{Resumen de la evidencia}

El oxígeno se ha empleado desde hace mucho tiempo en el manejo de pacientes con enfermedades respiratorias crónicas, ya que disminuye la demanda ventilatoria, mejora la función cardiovascular, hematológica y muscular, por lo cual mejora la disnea, la limitación funcional e incluso la sobrevida de los pacientes con enfermedades respiratorias crónicas ${ }^{3,5-7}$. Sin embargo, los estudios han mostrado resultados discordantes, fundamentalmente por tener diversos diseños, poblaciones con diferentes niveles de daño funcional y especialmente por no tener grupo control, como señalan Brusasco ${ }^{8}$ y Nonoyama ${ }^{9}$.

Lo que es claro hoy en día es que los pacientes con EPOC que tienen hipoxemia de reposo y/o que están recibiendo oxígenoterapia tendrían que utilizar oxígeno durante la RR, por cuanto hay evidencia que mejora significativamente la tolerancia al ejercicio y también disminuye la disnea ${ }^{1,3}$. No obstante, es muy probable que necesiten aumentar el flujo de oxígeno durante el período de entrenamiento muscular para no caer en niveles de hipoxemia. Esto quedó demostrado en el estudio de Dreher y cols ${ }^{10}$, en el cual los pacientes con EPOC severa durante un ejercicio físico (caminata) utilizaron el mismo flujo de oxígeno que usaban en sus domicilios y encontraron una caída de la $\mathrm{PaO}_{2}$ en promedio de $10 \mathrm{mmHg}$, varios de ellos con valores de $\mathrm{PaO}_{2}$ menores de $60 \mathrm{mmHg}$ (Figura 1).

Sin embargo, existe controversia en utilizar suplementación de oxígeno en paciente con EPOC sin hipoxemia de reposo. Al respecto se han estudiado dos poblaciones diferentes de pacientes: aquellos con hipoxemia inducida por el ejercicio y sin hipoxemia durante el ejercicio.

Con respecto a los pacientes con EPOC que presentan hipoxemia inducida por el ejercicio, hay pocos estudios que evalúen el beneficio de oxígeno suplementario durante la rehabilitación respiratoria. Rooyackers y cols ${ }^{11}$, realizaron un estudio en 24 pacientes con EPOC e hipoxemia inducida por ejercicio. Todos fueron incluidos en un programa de RR de diez semanas de duración y se asignó al azar a entrenamiento con oxígeno suplementario con $4 \mathrm{~L} / \mathrm{min}$ por naricera o aire ambiente. Ellos encontraron que la suplementación de oxígeno durante el entrenamiento no 


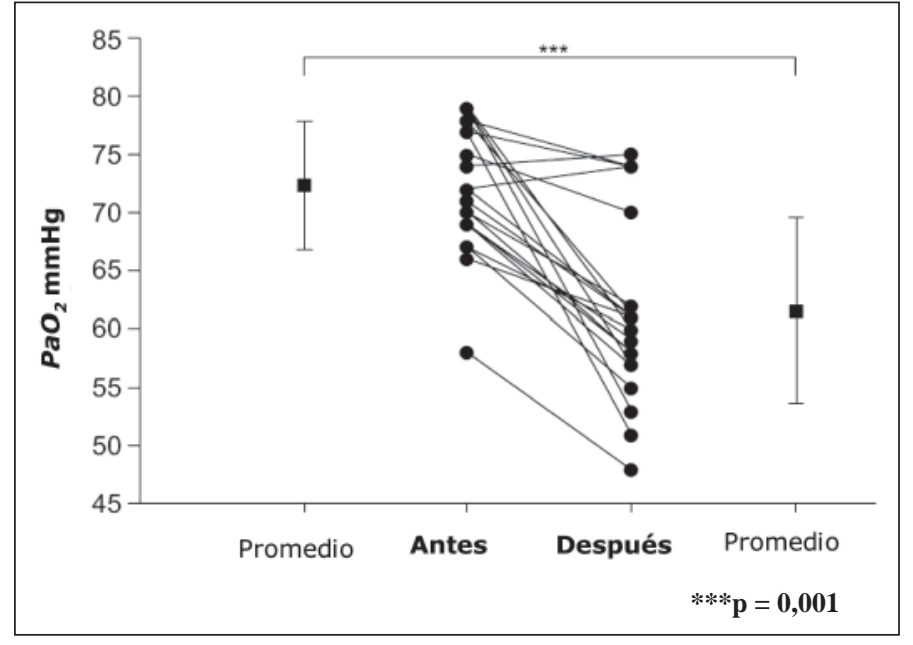

Figura 1. Pacientes con EPOC severa que durante la caminata utilizaron el mismo flujo de oxígeno que usaban en sus domicilios ${ }^{10}$. Después del ejercicio se observa una caída significativa de la $\mathrm{PaO}_{2}$ en promedio de 10 mmHg. Figura modificada del estudio de Dreher $\mathrm{M}$, et $\mathrm{al}^{10}$. aportó beneficios comparado con el aire ambiente, en cambio, la rehabilitación respiratoria mejora el rendimiento físico y la calidad de vida en ambos grupos de pacientes. Wadell y cols ${ }^{12}$, evaluaron en un estudio aleatorio-controlado, a 20 pacientes con EPOC y con hipoxemia de ejercicio. El entrenamiento físico fue realizado con oxígeno $5 \mathrm{~L} / \mathrm{min}(\mathrm{n}=10)$ y sin oxígeno $(\mathrm{n}=$ 10). Ellos concluyeron que la suplementación de oxígeno no mejoró significativamente el efecto del entrenamiento, en comparación con el grupo que fue entrenado con aire ambiente. Garrod y $\mathrm{cols}^{13}$, realizaron un estudio randomizado con 25 pacientes con EPOC severa $\left(\mathrm{VEF}_{1}=30 \%\right)$ que no tenían hipoxemia de reposo, pero desaturaban durante el ejercicio. Los pacientes entraron en un programa de RR y fueron asignados al azar a un grupo que recibió oxígeno $4 \mathrm{~L} / \min (\mathrm{n}=13)$ o al grupo sin suplemento de oxígeno $(\mathrm{n}=12)$. Ellos concluyen que el oxígeno suplementario durante el entrenamiento muscular tiene un pequeño efecto beneficioso en mejorar la capacidad de ejercicio y disminuir la disnea.

En relación al segundo grupo de pacientes, que no desarrollan hipoxemia con el ejercicio, un reciente estudio de Emtner y cols ${ }^{14}$, fue realizado en pacientes con EPOC grave $\left(\mathrm{VEF}_{1}\right.$ promedio de 36\%). Se utilizó para el estudio un diseño aleatorio-controlado, doble ciego. Los pacientes realizaban ejercicios de alta intensidad, bajo supervisión en un cicloergómetro durante 21 sesiones a lo largo de 7 semanas. Un grupo recibió oxígeno a $3 \mathrm{~L} / \mathrm{min}(\mathrm{n}=15)$ y el grupo control aire comprimido a $3 \mathrm{~L} / \mathrm{min}(\mathrm{n}=15)$. Los autores encuentran que el entrenamiento con oxígeno suplementario permitió realizar ejercicios de una mayor intensidad, mejorar el patrón respiratorio, y la capacidad de ejercicio comparado con el grupo control. Lo interesante de este estudio es que fue realizado en pacientes con EPOC sin hipoxemia de reposo ni durante el ejercicio.

Finalmente, Nonoyama y cols ${ }^{9}$, realizan una revisión sistemática y meta-análisis de la literatura. El objetivo del estudio fue determinar si el oxígeno suplementario en comparación con el control (aire comprimido o aire de la habitación) durante el entrenamiento muscular en un programa de rehabilitación pulmonar afecta la capacidad de realizar ejercicio, la magnitud de la disnea y la calidad de vida en pacientes con EPOC sin criterios de oxígenoterapia domiciliaria. De un total de 1.621 estudios revisados sólo cinco cumplían con los criterios de inclusión ${ }^{11-15}$, ya que debían ser estudios clínicos aleatorios y controlados. Ellos encuentran una mejoría significativa en los pacientes que recibieron oxígeno suplementario comparado con los controles con respecto a: 1) Tiempo de ejercicio, diferencias de medias ponderadas (DMP) 2,68 minutos (IC 95\%, 0,07 a 5,28 minutos; 2) Escala de Borg al final del ejercicio, DMP-1,22 unidades (IC 95, -2,39 a -0,06); 3) Cambio en la escala de Borg después de la prueba de caminata de carga progresiva, DMP $-1,46$ unidades (IC 95\%, -2,72 a -0,19). No hubo diferencias significativas en la capacidad máxima de ejercicio, prueba de caminata de 6 minutos, distancia en la prueba de caminata de carga progresiva, calidad de vida y en la oxigenación. De acuerdo con el sistema GRADE ${ }^{16}$, la mayoría de los resultados fueron clasificados como de baja calidad, ya que estaban limitados por la calidad de los estudios. Los autores concluyen que existe una pequeña y limitada evidencia a favor de la administración de oxígeno suplementario para el entrenamiento muscular de los pacientes con EPOC sin requerimientos de oxígeno en reposo. 


\section{Grado de recomendación}

Se recomienda utilizar suplemento de oxígeno en todo paciente con EPOC e hipoxemia de reposo durante el entrenamiento muscular de un programa de rehabilitación respiratoria (calidad de la evidencia A, recomendación fuerte). Así también, en los pacientes con EPOC y sin hipoxemia de reposo se recomienda utilizar suplemento de oxígeno durante la rehabilitación respiratoria, por cuanto permite tolerar durante mayor tiempo el entrenamiento muscular y reduce la disnea (calidad de la evidencia $B$, recomendación débil).

\section{Conclusiones}

Existe evidencia que los pacientes con EPOC sin hipoxemia de reposo se benefician del uso de suplemento de oxígeno durante la rehabilitación respiratoria. Idealmente debe usarse oxígeno al $30 \%$ a través de una máscara con Venturi, para evitar pérdidas si el paciente es respirador bucal, o con naricera a 3-4 L/min si la ventilación es nasal. Controlar con oxímetro de pulso, para asegurar una saturación de oxígeno alrededor de 92 a 93\%. En los pacientes que utilizan oxígenoterapia domiciliaria el flujo debe incrementarse para evitar períodos de hipoxemia durante el entrenamiento muscular.

Son necesarios nuevos estudios con un mayor número de participantes y con un mejor diseño que permitan obtener mejores y más fuertes conclusiones.

\section{Bibliografía}

1.- RABE K F, HURD S, ANZUETO A, BARNES P J, BUIST S A, CALVERLEY P, et al. Global strategy for the diagnosis, management, and prevention of chronic obstructive pulmonary disease: GOLD executive summary. Am J Respir Crit Care Med 2007; 176: 532-55.

2.- DECRAMER M, DE BENEDETTO F, DEL PONTE A, MARINARI S. Systemic effects of COPD. Respir Med 2005; 99 (Suppl B): S3-S10.

3.- O'DONNELL D E, D'ARSIGNY C, WEBB K A. Effects of hyperoxia on ventilatory limitation during exercise in advanced chronic obstructive pulmonary disease. Am J Respir Crit Care Med 2001; 163: 892-8.

4.- SOMFAY A, PORSZASZ J, LEE S M, CASABURI R. Dose-response effect of oxygen on hyperinflation and exercise endurance in nonhypoxaemic COPD patients. Eur Respir J 2001; 18: 77-84.

5.- MEDICAL RESEARCH COUNCIL WORKING PARTY. Long-term domiciliary oxygen therapy in chronic hypoxic cor pulmonale complicating chronic bronchitis and emphysema. Lancet 1981; 1 (8222): 681-6.
6.- Nocturnal Oxygen Therapy Trial group. Continuous or nocturnal oxygen therapy in hypoxemic chronic obstructive lung disease: a clinical trial. Ann Intern Med 1980; 93: 391-8.

7.- TARPY S P, CELLI B R. Long-term oxygen therapy. N Engl J Med 1995; 333: 710-4.

8.- BRUSASCO V, PELLEGRINO R. Oxygen in the rehabilitation of patients with chronic obstructive pulmonary disease: an old tool revisited. Am J Respir Crit Care Med 2003; 168: 1021-2.

9.- NONOYAMA M L, BROOKS D, LACASSE Y, GUYATT G H, GOLDSTEIN R S. Oxygen therapy during exercise training in chronic obstructive pulmonary disease. Cochrane Database Syst Rev 2007; 18 (2): CD005372.

10.- DREHER M, STORRE J H, WINDISCH W. Noninvasive ventilation during walking in patients with severe COPD: a randomised cross-over trial. Eur Respir J 2007; 29: 930-6.

11.- ROOYACKERS J M, DEKHUIJZEN P N, VAN HERWAARDEN C L, FOLGERING H T. Training with supplemental oxygen in patients with COPD and hypoxaemia at peak exercise. Eur Respir J 1997; 10: 1278-84.

12.- WADELL K, HENRIKSSON-LARSEN K, LUNDGREN R. Physical training with and without oxygen in patients with chronic obstructive pulmonary disease and exercise-induced hypoxaemia. J Rehabil Med 2001; 33: 200-5.

13.- GARROD R, PAUL E A, WEDZICHA J A. Supplemental oxygen during pulmonary rehabilitation in patients with COPD with exercise hypoxaemia. Thorax 2000; 55: 539-43.

14.- EMTNER M, PORSZASZ J, BURNS M, SOMFAY A, CASABURI R. Benefits of supplemental oxygen in exercise training in nonhypoxemic chronic obstructive pulmonary disease patients. Am J Respir Crit Care Med 2003; 168: 1034-42.

15.- FICHTER J, FLECKENSTEIN J, STAHL C, SYBRECHT G W. Effect of oxygen (FI02: 0.35) on the aerobic capacity in patients with COPD. Pneumologie 1999; 53: 121-6.

16.- SCHÜNEMANN H J, JAESCHKE R, COOK D J, BRIA W F, EL-SOLH A A, ERNST A, et al; ATS Documents Development and Implementation Committee. An official ATS statement: grading the quality of evidence and strength of recommendations in ATS guidelines and recommendations. Am J Respir Crit Care Med 2006; 174: 605-14.

Correspondencia a:

Dr. Juan Céspedes G.

Laboratorio de Función Pulmonar, Instituto Nacional del Tórax.

Av. J. M. Infante 717, Providencia. Santiago, Chile.

E-mail: jmcespedes@hotmail.com 